\title{
A Generalized Quantum Theory
}

\author{
Gerd Niestegge \\ Fraunhofer ESK, Hansastr. 32, 80686 München, Germany \\ gerd.niestegge@esk.fraunhofer.de, gerd.niestegge@web.de
}

\begin{abstract}
In quantum mechanics, the selfadjoint Hilbert space operators play a triple role as observables, generators of the dynamical groups and statistical operators defining the mixed states. One might expect that this is typical of Hilbert space quantum mechanics, but it is not. The same triple role occurs for the elements of a certain ordered Banach space in a much more general theory based upon quantum logics and a conditional probability calculus (which is a quantum logical model of the Lüders-von Neumann measurement process). It is shown how positive groups, automorphism groups, Lie algebras and statistical operators emerge from one major postulate - the non-existence of third-order interference (third-order interference and its impossibility in quantum mechanics were discovered by R. Sorkin in 1994). This again underlines the power of the combination of the conditional probability calculus with the postulate that there is no third-order interference. In two earlier papers, its impact on contextuality and nonlocality had already been revealed.
\end{abstract}

Key Words. Foundations of quantum mechanics, dynamical groups, positive groups, Lie algebras, operator algebras

PACS. 03.65Fd, 03.65:Ta, 02.30.Tb

\section{Introduction}

In quantum mechanics, the selfadjoint Hilbert space operators play a triple role. First of all, they represent the observables which are the physically measurable quantities of the system under consideration. Second, the normalized positive trace-class operators are called statistical operators; they define the mixed states of the system. Third, the selfadjoint operators are generators of one-parameter dynamical groups describing reversible time evolutions of the system.

One might expect that this triple role is typical of Hilbert space quantum mechanics. In the present paper, it will be shown that this is not true. Such a triple role occurs in a much more general theory based upon quantum logics and a conditional probability calculus which is a quantum logical model of the Lüders-von Neumann measurement process. This theory has been elaborated by the author in some recent papers $[9,10,11,12,13,14$.

A further major assumption is required; this is the absence of third-order interference. The concept of third-order interference was introduced by Sorkin who also recognized that third-order interference is ruled out by quantum me- 
chanics [15]. His concept was adapted to conditional probabilities by Barnum, Emerson and Ududec [16.

This paper does not consider the role of the observables in the generalized quantum theory, since it was already sufficiently studied in [10. The statistical operators are addressed briefly. The paper focuses on the group generators, using the theory of order derivations introduced by Connes [5].

In sections 2 and 3, the conditional probability calculus and Sorkin's concept of third-order interference are recapped as far as needed in this paper. First new results concerning the statistical operators and trace states are presented in section 4 . Order derivations are briefly sketched in section 5, and positive semigroups are considered in section 6 , before then turning to the major new results. These are the dynamical groups and Lie algebras emerging when thirdorder interference is ruled out (sections $7,8,9$ ). Section 10 is dedicated to equivalent reformulations of some of the mathematical conditions used so far by means of the conditional probabilities; this makes them accessible to physical interpretation. In the last two sections, it is shown how Jordan algebras and von Neumann algebras fit in the generalized theory.

\section{The conditional probability calculus}

In quantum mechanics, the measurable quantities of a physical system are represented by observables. Most simple are those observables where only the two discrete values 0 and 1 are possible as measurement outcome; these observables are called events (or propositions) and are elements of a mathematical structure called quantum logic.

A quantum logic $E$ contains two specific elements 0 and $\mathbb{I}$ and possesses an orthogonality relation $\perp$, an orthocomplementation $E \ni e \rightarrow e^{\prime} \in E$ and a partial sum operation + which is defined only for orthogonal events. Moreover, $e^{\prime} \perp e$ and $e+e^{\prime}=\mathbb{I}$ for $e \in E$. The interpretation of this mathematical terminology is as follows: orthogonal events are exclusive, $e^{\prime}$ is the negation of $e$, and $e+f$ is the conjunction or and-function of the two exclusive events $e$ and $f$.

The states on a quantum logic are the analogue of the probability measures in classical probability theory, and conditional probabilities can be defined similar to their classical prototype [9, 10, 11. A state $\mu$ allocates the probability $\mu(f) \in[0,1]$ to each event $f$, is additive for orthogonal events, and $\mu(\mathbb{I})=1$. The conditional probability of an event $f$ under another event $e$ is the updated probability for $f$ after the outcome of a first measurement has been the event $e$; it is denoted by $\mu(f \mid e)$. Mathematically, it is defined by the conditions that the map $E \ni f \rightarrow \mu(f \mid e)$ is a state on $E$ and that the identity $\mu(f \mid e)=\mu(f) / \mu(e)$ holds for all events $f \in E$ with $f \perp e^{\prime}$. It must be assumed that $\mu(e) \neq 0$.

However, among the abstractly defined quantum logics, there are many where no states or no conditional probabilities exist, or where the conditional probabilities are ambiguous. Therefore, only those quantum logics where sufficiently many states and unique conditional probabilities exist can be considered a satisfying framework for a probabilistic theory. 
In [10, 12, it has been shown that such a quantum logic $E$ generates an order-unit space $A$ (partially ordered real linear space with a specific norm; see [1]) and can be embedded in its unit interval $[0, \mathbb{I}]:=\{a \in A: 0 \leq a \leq \mathbb{I}\}$ $=\{a \in A: 0 \leq a$ and $\|a\| \leq 1\} ; \mathbb{I}$ becomes the order-unit, and $e^{\prime}=\mathbb{I}-e$ for $e \in E$. Each state $\mu$ on $E$ has a unique positive linear extension on $A$ which is again denoted by $\mu$.

Let $K$ denote the state space consisting of all states of the quantum logic $E$, and let $V$ be the real-linear space generated by $K$. A norm can be defined on $V$ such that $V$ becomes a base-norm space. The order-unit space $A$ is the dual of $V$, and the unit ball of $A$ is compact with regard to the weak topology $w(A, V) . A$ is the weakly closed linear hull of $E$.

Note below that an operator $S: A \rightarrow A$ on the order-unit space $A$ is called positive if $S(a) \geq 0$ for all $a$ in $A$ with $a \geq 0$. Most interesting are the positive operators $S$ with $S(\mathbb{I})=\mathbb{I}$; the reason is that, in this case, the map $E \ni e \rightarrow$ $\mu(S(e))$ defines a state $\mu S$ on $E$ for any state $\mu$ on $E$, and the map $S^{*}: \mu \rightarrow \mu S$ becomes a transformation of the state space $K$.

As shown in [10, 12, for each event $e$ in $E$, there is a weakly continuous positive linear operator $U_{e}: A \rightarrow A$ with the following properties: $\mu(f \mid e) \mu(e)=$ $\mu\left(U_{e} f\right)$ for all $f \in E$ and all states $\mu, \mu\left(U_{e} x\right)=\mu(x)$ for all $x \in A$ and any state $\mu$ with $\mu(e)=1, \mu\left(U_{e} x\right)=0$ for all $x \in A$ and any state $\mu$ with $\mu(e)=0$, $U_{e}^{2}=U_{e}, e=U_{e} e=U_{e} \mathbb{I}, 0=U_{e} f$ as well as $U_{e} U_{f}=0$ for $f \in E$ with $e \perp f$, and $f=U_{e} f$ for $e^{\prime} \perp f$.

These positive projections $U_{e}$ have many similarities with the compressions considered by Alfsen and Shultz 2] and called P-projections in their earlier papers. However, the two concepts differ; $U_{e} x=0$ with an event $e$ and a positive element $x$ in $A$ does not imply $U_{e^{\prime}} x=x$ and, therefore, $U_{e}$ is not a compression (P-projection). Moreover, Alfsen and Shultz's major interest are the spectral convex sets, but the results of this paper will show that a rich theory might also be possible without assuming spectrality.

Further weakly continuous linear operators $T_{e}$ and $S_{e}$ can now be defined for each $e \in E$ by $T_{e}(x):=\frac{1}{2}\left(x+U_{e} x-U_{e^{\prime}} x\right)$ and $S_{e}(x):=2 U_{e}(x)+2 U_{e^{\prime}} x-x$, $x \in A$. The properties of the operators $U_{e}$ above imply the following properties for these operators: $\mu\left(T_{e} x\right)=\mu(x)$ for all $x \in A$ and any state $\mu$ with $\mu(e)=1$, and $\mu\left(T_{e} x\right)=0$ for all $x \in A$ and any state $\mu$ with $\mu(e)=0$. Moreover, $e=T_{e} e=T_{e} \mathbb{I}, 0=T_{e} f$ for $f \in E$ with $e \perp f, S_{e}^{2} x=x$ for any $x$ in $A$, $T_{e}+S_{e} T_{e}=2 U_{e}$ and $U_{e}=2 T_{e}^{2}-T_{e}(e \in E)$.

In the remaining part of this paper, it shall always be assumed that $E$ is a quantum logic with the conditional probability calculus as described in this section. An interesting link between the linear operators $T_{e}$ and Sorkin's concept of third-order interference shall be considered in the following section. 


\section{Third-order interference}

Sorkin [15] introduced the following mathematical term $I_{3}$ for a triple of pairwise orthogonal events $e_{1}, e_{2}$ and $e_{3}$, a further event $f$ and a state $\mu$ :

$$
\begin{aligned}
I_{3}:= & \mu\left(f \mid e_{1}+e_{2}+e_{3}\right) \mu\left(e_{1}+e_{2}+e_{3}\right)-\mu\left(f \mid e_{1}+e_{2}\right) \mu\left(e_{1}+e_{2}\right) \\
& -\mu\left(f \mid e_{1}+e_{3}\right) \mu\left(e_{1}+e_{3}\right)-\mu\left(f \mid e_{2}+e_{3}\right) \mu\left(e_{2}+e_{3}\right) \\
& +\mu\left(f \mid e_{1}\right) \mu\left(e_{1}\right)+\mu\left(f \mid e_{2}\right) \mu\left(e_{2}\right)+\mu\left(f \mid e_{3}\right) \mu\left(e_{3}\right)
\end{aligned}
$$

He recognized that $I_{3}=0$ is universally valid in quantum mechanics. His original definition refers to probability measures on 'sets of histories'. Using conditional probabilities, $I_{3}$ gets the above shape, which was seen by Ududec, Barnum and Emerson [16].

For the three-slit set-up considered by Sorkin, the identity $I_{3}=0$ means that the interference pattern observed with three open slits is a simple combination of the patterns observed in the six different cases when only one or two of the three slits are open. The new type of interference which is present whenever $I_{3} \neq 0$ holds is called third-order interference.

In Ref. 12, it has been shown that the quantum logic $E$ rules out thirdorder interference $\left(I_{3}=0\right)$ if and only if the identity $T_{e+f} x=T_{e} x+T_{f} x$ holds for all orthogonal event pairs $e$ and $f$ in $E$ and all $x$ in $A$. Mathematically, this orthogonal additivity of $T_{e}$ in $e$ is a lot easier to handle than the equivalent identity $I_{3}=0$ with the above definition of the rather intricate term $I_{3}$ which, however, may be more meaningful physically.

Quantum logics which do not exhibit third-order interference (i.e., which satisfy the identity $I_{3}=0$ ) have been studied in Ref. [12, and it has been shown that there is a product operation $\square$ in the order-unit space $A$ generated by such a quantum logic, if the $\epsilon$-Hahn-Jordan decomposition property holds in addition ([12] Lemma 10.2).

The quantum logic $E$ is said to possess the $\epsilon$-Hahn-Jordan decomposition property if, for every bounded orthogonally additive real-valued function $\rho$ on $E$ and every $\epsilon>0$, there are two states $\mu$ and $\nu$, nonnegative real numbers $s$ and $t$ and an event $e$ in $E$ such that $\rho=s \mu-t \nu$ and $\mu(e)<\epsilon$ as well as $\nu\left(e^{\prime}\right)<\epsilon$. It implies that $[0, \mathbb{I}]$ is the weakly closed convex hull of $E[12$.

The product $a \square b$ is linear and weakly continuous in $a$ as well as in $b$ and satisfies the inequality $\|a \square b\| \leq\|a\|\|b\|(a, b \in A)$, where \|\| denotes the orderunit norm on $A$. For any events $e$ and $f$ in $E$, the identity $T_{e} f=e \square f$ holds. The events $e$ become idempotent elements in $A$ (i.e., $e=e^{2}=e \square e$ ), and $e \square f=0$ for any orthogonal event pair $e$ and $f$. Generally, however, the product is neither commutative nor associative. Moreover, the square $a^{2}=a \square a$ of an element $a$ in $A$ need not be positive. 


\section{Statistical operators}

An element $\mu \in K$ is called a trace state if $\mu(f)=\mu(f \mid e) \mu(e)+\mu\left(f \mid e^{\prime}\right) \mu\left(e^{\prime}\right)$ holds for all events $e, f$ in $E$. This means that all events are compatible under $\mu$ 11. With the linear operators $U_{e}, T_{e}$ and $S_{e}$ defined in section 2, it follows that $\mu$ is invariant under $U_{e}+U_{e^{\prime}}$ and $S_{e}$ for each event $e$ in $E$. The identity $T_{e}+S_{e} T_{e}=2 U_{e}$ then gives $\mu\left(T_{e} f\right)=\mu\left(U_{e} f\right) \geq 0$ for any events $e, f$ in $E$.

Suppose now that the $\epsilon$-Hahn-Jordan decomposition property and $I_{3}=0$ hold. For the then existing product and a trace state $\mu$, we get $\mu(e \square f)=$ $\mu\left(T_{e} f\right) \geq 0$ for any events $e$ and $f$, and therefore $\mu(x \square y) \geq 0$ for any positive elements $x$ and $y$ in $A$. Note that the $\epsilon$-Hahn-Jordan decomposition property implies that $[0, \mathbb{I}]$ is the weakly closed convex hull of $E[12$.

With a trace state $\mu$, each positive element $x$ in $A$ with $\mu(x)=1$ then gives rise to two further states: $e \rightarrow \mu(x \square e)$ and $e \rightarrow \mu(e \square x)$. They become identical if the product is commutative. Via this construction, the positive elements of the order unit space $A$ define states in the same way as the statistical operators do in Hilbert space quantum mechanics. An important question then becomes whether a trace state exists; this shall now be addressed.

Assume that the linear operator $S_{e}$ is positive for every event $e$ in $E$. Then its inverse $S_{e}^{-1}=S_{e}$ is positive, $S_{e}(\mathbb{I})=\mathbb{I}$, and these operators generate a positive group leaving $\mathbb{I}$ invariant. It shall now be seen that the assumed positivity of the $S_{e}$ has an important consequence: the existence of a trace state - at least in the finite-dimensional case. The following lemma from [4] will be used.

Lemma 1. Let $C$ be a compact convex set in a finite dimensional real-linear space. Then the group of all affine homeomorphisms of $C$ onto $C$ has a common fix point.

Theorem 1. Suppose that $S_{e}$ is positive for each event $e$ in $E$ and that the dimension of the order unit space $A$ is finite. Then a trace state exists on $E$.

Proof. Recall that $K$ is the state space of $E$, that $V$ is the linear space generated by $K$ and that $A$ is the dual space of $V$. Since $A$ has a finite dimension, so does $V$ and Lemma 1 can be applied to the compact convex set $K$. Therefore, there is a common fix point $\mu$ of the affine homeomorphisms of $K$.

Since the operators $S_{e}$ are positive and satisfy $S_{e}(\mathbb{I})=\mathbb{I}$, the transformations $S_{e}^{*}$ (defined in section 2) map states to states and thus define affine homeomorphisms of $K$. Therefore, the fix point $\mu$ is invariant under each $S_{e}$ : $\mu\left(S_{e} x\right)=\mu(x)$ for any $e \in E$ and $x \in A$. Reconsidering the definition of $S_{e}$ in section 2, this means that $\mu\left(U_{e} f\right)+\mu\left(U_{e^{\prime}} f\right)=\mu(f)$ for any events $e$ and $f$, and thus $\mu$ is a trace state.

Note that Theorem 1 does not require the assumptions that $I_{3}=0$ and the $\epsilon$-Hahn-Jordan decomposition property hold. 


\section{Group generators}

A bounded linear operator $D: A \rightarrow A$ is called an order dissipation, if $e^{t D}$ is positive for any $t \geq 0$, and is called an order derivation, if $e^{t D}$ is positive for any real number $t$. The order dissipations are generators of positive semigroups; each group element has an inverse which is a linear operator, but need not be positive. The order derivations are generators of positive groups; in this case, each group element has a positive inverse.

As described in section 2, the positive operators which map $\mathbb{I}$ to $\mathbb{I}$ give rise to transformations of the state space. Therefore, most interesting are those positive groups, which leave the order-unit invariant for all $t$; this holds when the generator $D$ satisfies the condition $D(\mathbb{I})=0$. Such a derivation $D$ generates a one-parameter group of automorphisms. It describes the dynamical evolution satisfying the simple linear differential equation $\frac{d}{d t} x_{t}=D x_{t}\left(x_{t} \in A\right)$. Any physical theory with a reversible time evolution should include such oneparameter automorphism groups and therefore at least some derivations $D$ with $D(\mathbb{I})=0$. Generally, they need not be bounded, but note that only bounded derivations are considered in this paper.

The following two lemmas provide useful characterizations of the order dissipations and order derivations; the first one is a result in [6] and implies the second one which can be found also in [1].

Lemma 2. Let $D: A \rightarrow A$ be a bounded linear operator. Then the following are equivalent:

(i) $D$ is an order dissipation.

(ii) If $0 \leq x \in A, \mu \in K$ and $\mu(x)=0$, then $\mu(D x) \geq 0$.

Lemma 3. Let $D: A \rightarrow A$ be a bounded linear operator. Then the following are equivalent:

(i) $D$ is an order derivation.

(ii) If $0 \leq x \in A, \mu \in K$ and $\mu(x)=0$, then $\mu(D x)=0$.

\section{Positive semigroups}

Now let $P: A \rightarrow A$ be a positive linear operator. Then $P^{n}$ is positive for $n=1,2,3, \ldots$ and, with $P^{0}=I$ and $I(x):=x$ for $x$ in $A$,

$$
e^{-t} \Sigma_{n=0}^{\infty} \frac{t^{n}}{n !} P^{n}=e^{-t} e^{t P}=e^{t(P-I)}
$$

is a convex combination of $I$ and $P^{n}(n=1,2,3, \ldots)$ for any $t \geq 0$ and therefore positive. This means that $P-I$ is an order dissipation. This also follows from Lemma 2.

Therefore, with any $e \in E, D:=U_{e}+U_{e^{\prime}}-I$ is an order dissipation with $D(1)=0$. Since $U_{e}+U_{e^{\prime}}$ is idempotent, $e^{t D}$ is a simple convex combination of $I$ and $U_{e}+U_{e^{\prime}}$ and this case is rather trivial. More interesting is $D:=\left(U_{e}+\right.$ $\left.U_{e^{\prime}}\right)\left(U_{f}+U_{f^{\prime}}\right)-I$ with a pair of events $e$ and $f$. If the linear operators $U_{e}$ and 
$U_{e^{\prime}}$ commute with $U_{f}$ and $U_{f^{\prime}}$, the product $\left(U_{e}+U_{e^{\prime}}\right)\left(U_{f}+U_{f^{\prime}}\right)$ is an idempotent operator again, yielding the same trivial situation as above. However, if they don't commute, this results in a non-trivial positive semigroup which leaves the order-unit invariant. Note that, in the classical case, $U_{e}+U_{e^{\prime}}=I=U_{f}+U_{f^{\prime}}$, $D=0, e^{t D}=I$ for any $t$, and the above construction becomes meaningless.

In the following two sections, it will be seen how the more interesting positive groups, automorphism groups and their Lie algebras emerge from the absence of third-order interference in the non-classical case.

\section{Positive groups}

In this section, it is assumed that $E$ rules out third-order interference $\left(I_{3}=\right.$ 0 ) and satisfies the $\epsilon$-Hahn-Jordan decomposition property. Then there is a product operation $\square$ in $A$ which is neither associative nor commutative in the general case. It will be seen now that, in many cases, the right-hand side multiplication operators $R_{a}: A \rightarrow A, R_{a}(x):=x \square a$ are order derivations generating positive groups $(a \in A)$.

One further assumption is required. Note that an element in a convex set is an extreme point of this set if it is not any convex combination of two other elements in this set. Denote by $\operatorname{ext}[0, \mathbb{I}]$ the set of extreme points of $[0, \mathbb{I}]$. For $\mu \in K$ and $e \in E$ with $\mu(e)=0$, we have from section 2 that $\mu(e \square x)=\mu\left(T_{e} x\right)=$ 0 for all $x \in A$. It shall now be assumed that this holds not only for the $e \in E$ with $\mu(e)=0$, but for the $e \in \operatorname{ext}[0, \mathbb{I}]$ with $\mu(e)=0$.

Theorem 2. Assume that $\mu(e \square x)=0$ for all $x \in A$, if $e \in \operatorname{ext}[0, \mathbb{I}]$ and $\mu \in K$ with $\mu(e)=0$. Then $R_{a}$ is an order derivation for any $a$ in $A$.

Proof. Suppose $a \in A$ and $\mu \in K$. Using Lemma 3, it is sufficient to show that

$$
\{x \in[0, \mathbb{I}]: \mu(x)=0\} \subseteq\left\{x \in[0, \mathbb{I}]: \mu\left(R_{a} x\right)=0\right\} .
$$

Both sets are convex and weakly compact and, by the Krein-Milman theorem, they are the closed convex hulls of their extreme points. Therefore, it is sufficient to show that any extreme point of the first set lies in the second one.

For any extreme point $e$ of the first set, suppose that $e=s b_{1}+(1-s) b_{2}$ with $0<s<1$ and $b_{1}, b_{2} \in[0, \mathbb{I}]$. Then $\mu(e)=0$ implies $\mu\left(b_{1}\right)=\mu\left(b_{2}\right)=0$ and both $b_{1}$ and $b_{2}$ lie in the first set. Since $e$ is an extreme point of this set, it follows that $e=b_{1}=b_{2}$. Therefore, $e$ is an extreme point of the unit interval, thus $\mu\left(R_{a} e\right)=\mu(e \square a)=0$, which means that $e$ lies in the second set.

\section{Automorphism groups}

Most interesting are the order derivations $D$ with $D(\mathbb{I})=0$, since then the positive groups they generate leave the order-unit $\mathbb{I}$ invariant and give rise to transformation groups of the state space. This case shall now be studied. Assume again that $E$ rules out third-order interference $\left(I_{3}=0\right)$, that $E$ satisfies 
the $\epsilon$-Hahn-Jordan decomposition property and that $\mu(e \square x)=0$ for all $x \in A$, if $e \in \operatorname{ext}[0, \mathbb{I}]$ and $\mu \in K$ with $\mu(e)=0$.

An order derivation $D$ is called skew, if $D(\mathbb{I})=0$, and is called selfadjoint, if there is an element $a \in A$ with $D=R_{a}$. Of course, $R_{a}(\mathbb{I})=a$. This naming (selfadjoint and skew) is rather unmotivated here, but will become clear later when the von Neumann algebras will be considered as an example.

Any order derivation $D$ is the sum of a selfadjoint order derivation $D_{1}$ and a skew order derivation $D_{2}$; with $a:=D(\mathbb{I})$ choose $D_{1}:=R_{a}$ and $D_{2}:=D-D_{1}$. The commutator $D_{0}:=\left[D_{1}, D_{2}\right]=D_{1} D_{2}-D_{2} D_{1}$ of any two order derivations $D_{1}$ and $D_{2}$ is an order derivation again and the order derivations form a Lie algebra [1].

It is obvious that the commutator is skew if $D_{1}$ and $D_{2}$ are skew. Therefore the skew order derivations form a Lie subalgebra $L$ which shall be called the Lie algebra of the quantum logic $E$. Its elements are generators of one-parameter automorphism groups which describe reversible dynamical evolutions. With any pair of elements $a$ and $b$ in the order-unit space $A$, the operator $\left[R_{a}, R_{b}\right]-R_{d}$ with $d:=b \square a-a \square b$ now lies in the Lie algebra $L$ by Theorem 2 . The associativity of the product $\square$ would imply that $\left[R_{a}, R_{b}\right]-R_{d}=0$; however, it is not associative generally.

\section{The commutative case}

The question whether not only the right-hand side multiplication operators $R_{a}$, but also the left-hand side multiplication operators $T_{a}: A \rightarrow A, T_{a} x:=a \square x$ are order derivations for $a \in A$, shall now be addressed; it will turn out that they are so if and only if the product $\square$ is commutative. Note that these $T_{e}$ with $e \in E$ coincide with the linear operators $T_{e}$ considered in section 2 .

The following lemma holds under the general assumptions of section 2 and does not require the further assumptions concerning third-order interference, the Hahn-Jordan decomposition property and the extreme points of $[0, \mathbb{I}]$.

Lemma 4. If the operators $T_{e}-T_{e^{\prime}}$ and $T_{f}-T_{f^{\prime}}$ are order derivations for two events $e, f \in E$, then the identity $T_{e} f=T_{f} e$ holds.

Proof. Assume that $D_{e}:=T_{e}-T_{e^{\prime}}=U_{e}-U_{e^{\prime}}$ and $D_{f}:=T_{f}-T_{f^{\prime}}=U_{f}-U_{f^{\prime}}$ are order derivations for the two events $e, f \in E$, and define the positive operators $P_{e}:=U_{e}+U_{e^{\prime}}$ and $P_{f}:=U_{f}+U_{f^{\prime}}$. Note that $D_{e}^{2}=P_{e}$ and $D_{f}^{2}=P_{f}$.

Then $\mu\left(U_{f^{\prime}} U_{f} x\right)=0$ for any $\mu \in K$ and $0 \leq x \in A$, since $U_{f^{\prime}} U_{f}=0$. Applying Lemma 3 to the derivation $D_{e}$, the positive linear functional $\mu U_{f^{\prime}}$ and the positive element $U_{f} x$ in $A$, it follows that $\mu\left(U_{f^{\prime}} D_{e} U_{f} x\right)=0$ for any $\mu \in K$ and $0 \leq x \in A$. Therefore $U_{f^{\prime}} D_{e} U_{f}=0$. Similarly $U_{f} D_{e} U_{f^{\prime}}=0$. An immediate consequence is

$$
\left(U_{f}-U_{f^{\prime}}\right)\left(U_{e}-U_{e^{\prime}}\right)\left(U_{f}-U_{f^{\prime}}\right)=\left(U_{f}+U_{f^{\prime}}\right)\left(U_{e}-U_{e^{\prime}}\right)\left(U_{f}+U_{f^{\prime}}\right)
$$

(both sides being equal to $U_{f} D_{e} U f+U_{f^{\prime}} D_{e} U_{f^{\prime}}$ ). Clearly the same equality holds with exchanged roles of $e$ and $f$. Thus 


$$
\begin{aligned}
& D_{f} D_{e} D_{f}=P_{f} D_{e} P_{f}, D_{e} D_{f} D_{e}=P_{e} D_{f} P_{e} \text { and } \\
\left(D_{e} D_{f}-\right. & \left.D_{f} D_{e}\right)^{2} \mathbb{I} \\
= & D_{e} D_{f} D_{e} D_{f} \mathbb{I}+D_{f} D_{e} D_{f} D_{e} \mathbb{I}-D_{e} D_{f}^{2} D_{e} \mathbb{I}-D_{f} D_{e}{ }^{2} D_{f} \mathbb{I} \\
= & D_{e} D_{f} D_{e} D_{f} \mathbb{I}+D_{f} D_{e} D_{f} D_{e} \mathbb{I}-D_{e} P_{f} D_{e} \mathbb{I}-D_{f} P_{e} D_{f} \mathbb{I} \\
= & D_{e} D_{f} D_{e} D_{f} \mathbb{I}+D_{f} D_{e} D_{f} D_{e} \mathbb{I}-D_{e} P_{f} D_{e} P_{f} \mathbb{I}-D_{f} P_{e} D_{f} P_{e} \mathbb{I} \\
= & 0 .
\end{aligned}
$$

In the second but last line, the identities $P_{e} \mathbb{I}=\mathbb{I}=P_{f} \mathbb{I}$ have been used to replace $\mathbb{I}$ by $P_{e} \mathbb{I}$ and $P_{f} \mathbb{I}$, respectively, and the last line follows from the identity above. Therefore

$$
e^{t\left(D_{e} D_{f}-D_{f} D_{e}\right)} \mathbb{I}=\mathbb{I}+t\left(D_{e} D_{f}-D_{f} D_{e}\right) \mathbb{I} .
$$

Since the set of order derivations is closed under commutators [1], $D_{e} D_{f}-D_{f} D_{e}$ is an order derivation and the left-hand side of the last equation is positive for all $t$. This implies

$$
\begin{aligned}
0 & =\left(D_{e} D_{f}-D_{f} D_{e}\right) \mathbb{I} \\
& =\left(T_{e}-T_{e^{\prime}}\right)\left(f-f^{\prime}\right)-\left(T_{f}-T_{f^{\prime}}\right)\left(e-e^{\prime}\right) \\
& =\left(T_{e}-T_{e^{\prime}}\right)(2 f-\mathbb{I})-\left(T_{f}-T_{f^{\prime}}\right)(2 e-\mathbb{I}) \\
& =2 T_{e} f-2 T_{e^{\prime}} f-e+e^{\prime}-2 T_{f} e+2 T_{f^{\prime}} e+f-f^{\prime} \\
& =2 T_{e} f-2 f+2 T_{e} f-e+e^{\prime}-2 T_{f} e+2 e-2 T_{f} e+f-f^{\prime} \\
& =4 T_{e} f-4 T_{f} e .
\end{aligned}
$$

In the second but last line, the identities $T_{e}+T_{e^{\prime}}=I=T_{f}+T_{f^{\prime}}$ have been used. Therefore $T_{e} f=T_{f} e$.

Theorem 3. Suppose that $I_{3}=0$ and the $\epsilon$-Hahn-Jordan decomposition property hold and that $\mu(e \square x)=0$ for all $x \in A$, if $e \in \operatorname{ext}[0, \mathbb{I}]$ and $\mu \in K$ with $\mu(e)=0$. Then the following are equivalent:

(i) For any $a \in A$, the operator $T_{a}$ is an order derivation.

(ii) The product $\square$ is commutative.

Proof. Assume (i). Then particularly the operators $T_{e-e^{\prime}}=T_{e}-T_{e^{\prime}}$ are order derivations for the events $e$ in $E$, and Lemma 4 implies $T_{e} f=T_{f} e$ for any two events $e$ and $f$ in $E$. This means $e \square f=f \square e$. Since the product is linear and weakly continuous in each component and $A$ is the weakly closed linear hull of $E$, the product is commutative.

Now assume (ii). This means that $T_{a}=R_{a}$ for $a \in A$, and the $T_{a}$ become order derivations by Theorem 2 . 
If the product $\square$ is commutative, the commutator $\left[R_{a}, R_{b}\right]=\left[T_{a}, T_{b}\right]$ is a skew order derivation for any two elements $a$ and $b$ in $A$ and thus lies in the Lie algebra $L$. In this case, $L=\{0\}$ would imply that the operators $T_{a}, a \in A$, and particularly the $T_{e}, e \in e$, commute with each other. Then the $U_{e}$ would commute and it would follow that, with any $e, f \in E, U_{e} f+U_{e^{\prime}} f=U_{e} U_{f} \mathbb{I}+$ $U_{e^{\prime}} U_{f} \mathbb{I}=U_{f} U_{e} \mathbb{I}+U_{f} U_{e^{\prime}} \mathbb{I}=U_{f} e+U_{f} e^{\prime}=U_{f} \mathbb{I}=f$. This would mean that all events in the quantum logic $E$ would be compatible [11] and $E$ would be classical. Vice versa, as soon as there are two events which are not compatible, the Lie algebra $L$ is not trivial.

\section{Some equivalent reformulations by means of the conditional probabilities}

The property that the operators $T_{e}-T_{e^{\prime}}$ are order derivations for all events $e$ has been studied by Iochum and Shultz under the name ellipticity in a more specific setting in order to characterize the state spaces of the JBW algebras among the spectral convex sets [2, 7]. The proof of Lemma 4 is a simple transfer of the proof of Theorem 9.48 in 2] to the more general setting of this paper.

Ellipticity is a mathematical property which has no immediate physical or probabilistic interpretation. The next lemma presents an equivalent property which is more accessible to interpretations.

Lemma 5. Under the general assumptions of section 2, the following are equivalent for any event $e$ in the quantum logic $E$ :

(i) $T_{e}-T_{e^{\prime}}$ is an order derivation.

(ii) $\mu(f)-\mu(f \mid e) \mu(e)-\mu\left(f \mid e^{\prime}\right) \mu\left(e^{\prime}\right) \geq-2 \sqrt{\mu(f \mid e) \mu(e) \mu\left(f \mid e^{\prime}\right) \mu\left(e^{\prime}\right)}$ for all events $f$ and all states $\mu$.

(iii) $\mu(f)-\mu(f \mid e) \mu(e)-\mu\left(f \mid e^{\prime}\right) \mu\left(e^{\prime}\right) \leq 2 \sqrt{\mu\left(f^{\prime} \mid e\right) \mu(e) \mu\left(f^{\prime} \mid e^{\prime}\right) \mu\left(e^{\prime}\right)}$ for all events $f$ and all states $\mu$.

Proof. (i) $\Leftrightarrow$ (ii): Note that $T_{e}-T_{e^{\prime}}=U_{e}-U_{e^{\prime}},\left(U_{e}-U_{e^{\prime}}\right)^{2}=U_{e}+U_{e^{\prime}}$ and $\left(U_{e}-U_{e^{\prime}}\right)\left(U_{e}+U_{e^{\prime}}\right)=U_{e}-U_{e^{\prime}}$. Therefore, $\left(U_{e}-U_{e^{\prime}}\right)^{n}=U_{e}-U_{e^{\prime}}$ for $n=1,3,5, \ldots,\left(U_{e}-U_{e^{\prime}}\right)^{n}=U_{e}+U_{e^{\prime}}$ for $n=2,4,6, \ldots$ and

$$
\begin{aligned}
\exp \left(t \left(T_{e}\right.\right. & \left.\left.-T_{e^{\prime}}\right)\right)=\sum_{n=0}^{\infty} \frac{t^{n}}{n !}\left(U_{e}-U_{e^{\prime}}\right)^{n} \\
& =I+\sum_{n=1}^{\infty} \frac{t^{n}}{n !} U_{e}+\sum_{n=1}^{\infty} \frac{(-t)^{n}}{n !} U_{e^{\prime}} \\
& =I+\sum_{n=0}^{\infty} \frac{t^{n}}{n !} U_{e}+\sum_{n=0}^{\infty} \frac{(-t)^{n}}{n !} U_{e^{\prime}}-U_{e}-U_{e^{\prime}} \\
& =I+\exp (t) U_{e}+\exp (-t) U_{e^{\prime}}-U_{e}-U_{e^{\prime}}
\end{aligned}
$$

for any real number $t$. Positivity of this operator means that

$$
0 \leq \mu\left(f+\exp (t) U_{e} f+\exp (-t) U_{e^{\prime}} f-U_{e} f-U_{e^{\prime}} f\right)
$$


for all events $f$ and states $\mu$. That is

$0 \leq \mu(f)+\exp (t) \mu(f \mid e) \mu(e)+\exp (-t) \mu\left(f \mid e^{\prime}\right) \mu\left(e^{\prime}\right)-\mu(f \mid e) \mu(e)-\mu\left(f \mid e^{\prime}\right) \mu\left(e^{\prime}\right)$.

Now note that, with any two nonnegative real numbers $\alpha$ and $\beta$, the largest lower bound for the function $t \rightarrow \alpha \exp (t)+\beta \exp (-t)$ is $2 \sqrt{\alpha \beta}$. Therefore, the last inequality holds for all real $t$ if and only if

$$
0 \leq \mu(f)+2 \sqrt{\mu(f \mid e) \mu(e) \mu\left(f \mid e^{\prime}\right) \mu\left(e^{\prime}\right)}-\mu(f \mid e) \mu(e)-\mu\left(f \mid e^{\prime}\right) \mu\left(e^{\prime}\right) .
$$

(ii) $\Leftrightarrow$ (iii): Replacing $f$ by $f^{\prime}$ in (ii) gives:

$1-\mu(f)-\mu(e)+\mu(f \mid e) \mu(e)-\mu\left(e^{\prime}\right)+\mu\left(f \mid e^{\prime}\right) \mu\left(e^{\prime}\right) \geq-2 \sqrt{\mu\left(f^{\prime} \mid e\right) \mu(e) \mu\left(f^{\prime} \mid e^{\prime}\right) \mu\left(e^{\prime}\right)}$

and $\mu(f)-\mu(f \mid e) \mu(e)-\mu\left(f \mid e^{\prime}\right) \mu\left(e^{\prime}\right) \leq 2 \sqrt{\mu\left(f^{\prime} \mid e\right) \mu(e) \mu\left(f^{\prime} \mid e^{\prime}\right) \mu\left(e^{\prime}\right)}$,

which completes the proof.

With Lemma 5, ellipticity becomes a feature of the conditional probabilities and imposes important restrictions on the typical quantum interference which is exhibited by the violation of the classical identity $\mu(f)=\mu(f \mid e) \mu(e)+\mu\left(f \mid e^{\prime}\right) \mu\left(e^{\prime}\right)$, $e, f \in E$ and $\mu \in K$. Lemma 5 (ii) and (iii) provide a lower bound and an upper bound for the interference term $\mu(f)-\mu(f \mid e) \mu(e)-\mu\left(f \mid e^{\prime}\right) \mu\left(e^{\prime}\right)$.

The other condition in Lemma 4 can also be equivalently reformulated by means of the conditional probabilities in the following way.

Lemma 6. Under the general assumptions of section 2 , the following are equivalent for any two events $e$ and $f$ in the quantum logic $E$ :

(i) $T_{e} f=T_{f} e$.

(ii) $\mu\left(f^{\prime} \mid e\right) \mu(e)+\mu\left(f \mid e^{\prime}\right) \mu\left(e^{\prime}\right)=\mu\left(e^{\prime} \mid f\right) \mu(f)+\mu\left(e \mid f^{\prime}\right) \mu\left(f^{\prime}\right)$ for all states $\mu$.

Proof. Condition (ii) means $U_{e} f^{\prime} U_{e^{\prime}} f=U_{f} e^{\prime}+U_{f^{\prime}} e$ and this is equivalent to $T_{e} f=T_{f} e$.

Under the assumptions of Theorem 3, condition (ii) or (iii) of Lemma 5 and condition (ii) of Lemma 6 become equivalent, since condition (i) of Lemma 5 and condition (i) of Lemma 6 are equivalent then. The equivalence of these properties of the conditional probabilities will be hard to see directly - without considering the order-unit space $A$ and the operators $U_{e}$ and $T_{e}$ on $A(e \in E)$. The same holds for the implications in the following corollary.

Corollary 1. Under the general assumptions of section 2, the quantum logic $E$ rules out third-order interference, whenever it satisfies one of the following four conditions:

(i) $T_{e}-T_{e^{\prime}}$ is an order derivation for each $e \in E$ (i.e., the state space $K$ is elliptic).

(ii) $\mu(f)-\mu(f \mid e) \mu(e)-\mu\left(f \mid e^{\prime}\right) \mu\left(e^{\prime}\right) \geq-2 \sqrt{\mu(f \mid e) \mu(e) \mu\left(f \mid e^{\prime}\right) \mu\left(e^{\prime}\right)}$ for all events $e, f \in E$ and all states $\mu \in K$. 
(iii) $\mu(f)-\mu(f \mid e) \mu(e)-\mu\left(f \mid e^{\prime}\right) \mu\left(e^{\prime}\right) \leq 2 \sqrt{\mu\left(f^{\prime} \mid e\right) \mu(e) \mu\left(f^{\prime} \mid e^{\prime}\right) \mu\left(e^{\prime}\right)}$ for all events $e, f \in E$ and all states $\mu \in K$.

(iv) $\mu\left(f^{\prime} \mid e\right) \mu(e)+\mu\left(f \mid e^{\prime}\right) \mu\left(e^{\prime}\right)=\mu\left(e^{\prime} \mid f\right) \mu(f)+\mu\left(e \mid f^{\prime}\right) \mu\left(f^{\prime}\right)$ for all events $e, f \in E$ and all states $\mu \in K$.

Proof. By Lemma 4, 5 and 6, each one of the conditions (i), (ii), (iii) and (iv) implies $T_{e} f=T_{f} e$ for any $e, f \in E$. Now suppose $e_{1}, e_{2} \in E$ with $e_{1} \perp e_{2}$. Then $T_{e_{1}+e_{2}} f=T_{f}\left(e_{1}+e_{2}\right)=T_{f} e_{1}+T_{f} e_{2}=T_{e_{1}} f+T_{e_{2}} f$ for all $f \in E$ and thus $T_{e_{1}+e_{2}} x=T_{e_{1}} x+T_{e_{2}} x$ for all $x \in A$. This is equivalent to $I_{3}=0$ for all states and events (see section 3).

The positivity of the operators $S_{e}, e \in E$, which plays an important role in section 4, shall now be reconsidered. Again there is an equivalent property of the conditional probabilities.

Lemma 7. Under the general assumptions of section 2 , the following are equivalent for any event $e$ in the quantum logic $E$ :

(i) $S_{e}$ is positive.

(ii) $\mu(f) \leq 2 \mu(f \mid e) \mu(e)+2 \mu\left(f \mid e^{\prime}\right) \mu\left(e^{\prime}\right)$ for all events $f$ and states $\mu$.

Proof. Condition (ii) means $I \leq 2\left(U_{e}+U_{e^{\prime}}\right)$. That is $0 \leq S_{e}$.

More information concerning the physical interpretation can be found in Refs. [1, 2, 12] for condition (ii) of Lemma 6 and in Ref. [12] for condition (ii) of Lemma 7.

\section{Jordan algebras and Lie algebras}

The formally real Jordan algebras were introduced by Jordan, von Neumann and Wigner [8]. Much later, this theory was extended to include infinite dimensional algebras; these are the so-called JB-algebras and JBW-algebras [2.

The idempotent elements of a JBW-algebra $A$ form a quantum logic $E$. In this case, $E=\operatorname{ext}[0, \mathbb{I}]$ holds. If the Jordan algebra does not contain a direct summand of type $I_{2}, E$ possesses a conditional probability calculus [9]. With the so-called triple product $\{x, y, z\}:=x \circ(y \circ z)-y \circ(z \circ x)+z \circ(x \circ y)$, then $U_{e} a=\{e, a, e\}, T_{e} a=e \circ a$, and $S_{e} a=\left\{e-e^{\prime}, a, e-e^{\prime}\right\}$ for any $a \in A$ and $e \in E$. The operators $S_{e}$ are positive.

Moreover, third-order interference is ruled out, and the $\epsilon$-Hahn-Jordan decomposition property is satisfied. The product $\square$ coincides with the Jordan product $\circ$ and is commutative. The order automorphisms leaving the unit $\mathbb{I}$ invariant coincide with the Jordan automorphisms. Each property of the conditional probabilities considered in section 10 is satisfied.

There are three classes of simple formally real Jordan algebras with finite dimension and one further case. These are the hermitian $n \times n$-matrices with real, complex and quaternion entries, equipped with the usual Jordan product, and the hermitian $3 \times 3$-matrices with octonion entries [2]. The Lie algebras 
of the quantum logics consisting of the idempotent elements of these Jordan algebras are $\mathfrak{s o}(n), \mathfrak{s u}(n), \mathfrak{s p}(n)$ and the exceptional Lie algebra $\mathfrak{f}_{4}$.

However, there are four further exceptional simple Lie algebras with finite dimension $\left(\mathfrak{g}_{2}, \mathfrak{e}_{6}, \mathfrak{e}_{7}\right.$ and $\left.\mathfrak{e}_{8}\right)$ to which no formally real Jordan algebra can be allocated [3]. An important question now becomes whether they are the Lie algebras of some unknown quantum logics. In the case of a positive answer, it would be interesting to study the characteristics which distinguish them from the quantum logics emerging from the formally real Jordan algebras. Considering the results in [10, 12], it is very likely that their state spaces are not spectral (in the meaning of Alfsen and Shultz [2]).

\section{Von Neumann algebras}

Quantum mechanics uses a very special type of quantum logic $E$; it consists of the selfadjoint projection operators on a Hilbert space or, more generally, in a von Neumann algebra $M$. The selfadjoint part of a von Neumann algebra $M$ is a JBW-algebra $A$. In this case, $U_{e} a=e a e, T_{e} a=(e a+a e) / 2, S_{e} a=$ $\left(e-e^{\prime}\right) a\left(e-e^{\prime}\right)$ and moreover $e^{t R_{b}} a=e^{t b / 2} a e^{t b / 2}(a, b \in A, e \in E)$. Again each property of the conditional probabilities considered in section 10 is satisfied.

Furthermore, $D_{b} a:=i(b a-a b) / 2(a \in A)$ defines a skew order derivation $D_{b}$ for any $b$ in $A$ and $e^{t D_{b}} a=e^{i t b / 2} a e^{-i t b / 2}(a \in A, t \in \mathbb{R})$. This specific relation between the elements of $A$ and skew order derivations distinguishes those JBW-algebras that are the selfadjoint part of a von Neumann algebras from the other JBW-algebras. Its mathematical formalization is the so-called dynamical correspondence 2. Generally, in a JBW algebra $A$, a skew order derivation $D$ can be derived via $D:=\left[T_{a}, T_{b}\right]$ from a pair $a$ and $b$ in $A$ which does not operator-commute, but not from a single element in $A$.

\section{Conclusions}

Those quantum logics that entail the conditional probability calculus appear to provide a promising generalized quantum theory. Many of its mathematical properties can be formulated by means of the conditional probabilities, which makes them accessible to physical interpretations.

In the present paper, it has been shown how dynamical groups and Lie algebras emerge when third-order interference is ruled out. This again underlines the power of the combination of the conditional probability calculus with the postulate that there is no third-order interference. Its impact on contextuality and nonlocality had already been revealed in two earlier papers [13, 14].

An interesting open question now becomes whether the four finite-dimensional simple exceptional real Lie algebras that do not arise from the automorphism groups of the formally really Jordan algebras perhaps arise from the automorphism groups of some unknown quantum logics. 


\section{References}

[1] Alfsen, E. M., and Shultz, F. W.: State spaces of operator algebras: basic theory, orientations and $\mathrm{C}^{*}$-products. Mathematics: Theory \& Applications, Birkhäuser Boston (2001)

[2] Alfsen, E. M., and Shultz, F. W.: Geometry of state spaces of operator algebras. Mathematics: Theory \& Applications, Birkhäuser Boston (2003)

[3] Baez, J. C.: The octonions. Bull. Amer. Math. Soc. 39, 145-205 (2001)

[4] Chu Cho-Ho and Wright, J. D. M.: A theory of types for convex sets and ordered Banach spaces. Proc. London Math. Soc. 36, 494-517 (1978)

[5] Connes, A.: Characterisation des espaces vectoriels ordonnes sousjacent aux algebres de von Neumann. Ann. Inst. Fourier (Grenoble) 24, 121-155 (1974)

[6] Evans, D. and Hanche-Olsen, H.: The generators of positive semigroups. J. Func. Analysis 32, 207-212 (1979)

[7] Iochum, B. and Shultz, F. W.: Normal state spaces of Jordan and von Neumann algebras. J. Func. Analysis 50, 317-328 (1983)

[8] Jordan, P., von Neumann, J. and Wigner, E.: On an algebraic generalization of the quantum mechanical formalism. Ann. Math. 35, 29-64 (1934)

[9] Niestegge, G.: Non-Boolean probabilities and quantum measurement. J. Phys. A 34, 6031-6042 (2001)

[10] Niestegge, G.: A representation of quantum mechanics in order-unit spaces. Found. Phys. 38, 783-795 (2008)

[11] Niestegge, G.: A hierarchy of compatibility and comeasurability levels in quantum logics with unique conditional probabilities. Commun. Theor. Phys. (Beijing, China) 54, 974-980 (2010)

[12] Niestegge, G.: Conditional probability, three-slit experiments, and the Jordan algebra structure of quantum mechanics. Adv. Math. Phys. 2012, 156573 (2012)

[13] Niestegge, G.: Three-slit experiments and quantum nonlocality. Found. Phys. 43, 805-812 (2013)

[14] Niestegge, G.: Super quantum probabilities and three-slit experiments Wright's pentagon state and the Popescu-Rohrlich box require third-order interference. Phys. Scr. T160, 014034 (2014)

[15] Sorkin, R. D.: Quantum mechanics as quantum measure theory. Mod. Phys. Lett. A 9, 3119-3127 (1994)

[16] Ududec, C., Barnum, H., and Emerson, J.: Three slit experiments and the structure of quantum theory. Found. Phys. 41, 396-405 (2011) 